\title{
Effect of Chlorhexidine Mouthrinse on Prevention of Microbial Contamination during EBUS-TBNA: A Study Protocol for a Randomized Controlled Trial
}

\author{
Na Young Kim, M.D. ${ }^{1}$ iD, Jae Hyeon Park, M.D. ${ }^{2,3}$, Jimyung Park, M.D. ${ }^{1}$, Nakwon Kwak, M.D. ${ }^{1,4}$, Sun \\ Mi Choi, M.D. ${ }^{1,4}$, Young Sik Park, M.D. ${ }^{1}$, Chang-Hoon Lee, M.D., Ph.D. ${ }^{1}$ and Jaeyoung Cho, M.D., \\ Ph.D. ${ }^{1,4}$ \\ ${ }^{\mathrm{I}}$ Division of Pulmonary and Critical Care Medicine, Department of Internal Medicine, ${ }^{2}$ Department of Laboratory Medicine, \\ Seoul National University Hospital, Seoul, Departments of ${ }^{3}$ Laboratory Medicine and ${ }^{4}$ Internal Medicine, Seoul National \\ University College of Medicine, Seoul, Republic of Korea
}

Background: Endobronchial ultrasound-guided transbronchial needle aspiration (EBUS-TBNA) is a standard diagnostic method for mediastinal and hilar lymphadenopathy. Although rare, fatal infectious complications can occur following EBUS-TBNA. However, to date, there is a lack of effective preventive strategies to reduce these complications. We started a trial to investigate the effect of chlorhexidine mouthrinse on the prevention of microbial contamination during EBUSTBNA.

Methods: This study is a single-center, parallel-group, assessor-blinded randomized controlled trial (RCT). We will enroll 112 adult participants undergoing EBUS-TBNA using a convex probe, and randomly assign them to two groups at a 1:1 ratio. The intervention group will gargle for 1 minute with $100 \mathrm{~mL}$ of $0.12 \%$ chlorhexidine gluconate before EBUSTBNA, while the control group will have no mouthrinse before the procedure. Immediately after completion of EBUSTBNA on all targeted lesions with an aspiration needle, a needle wash sample will be taken by instilling $5 \mathrm{~mL}$ of sterile saline into the used needle. The primary outcome is colony forming unit (CFU) counts in aerobic cultures of the needle wash samples. Secondary outcomes are CFU counts in anaerobic cultures, fever within 24 hours after EBUS-TBNA, and infectious complications within 4 weeks after EBUS-TBNA.

Conclusion: This trial was designed as the first RCT to investigate the effect of chlorhexidine mouthrinse on the prevention of microbial contamination during EBUS-TBNA. Results from this trial can provide clinical evidence for a simple, safe, and cost-effective strategy to prevent infectious complications following EBUS-TBNA (ClinicalTrials.gov ID: NCT04718922, registered on 22 January 2021).

Keywords: EBUS-TBNA; Infectious Complication; Chlorhexidine Gluconate; Mouthrinse

Address for correspondence: Jaeyoung Cho, M.D., Ph.D.

Department of Internal Medicine, Seoul National University College of Medicine, 101 Daehak-ro, Jongno-gu, Seoul 03080, Republic of Korea Phone: 82-2-2072-2503, Fax: 82-2-762-9662,E-mail: apricot6@snu.ac.kr

Received: Apr. 20, 2021, Revised: Jun. 3, 2021, Accepted: Jun. 23, 2021, Published online: Jun. 24, 2021

(a) It is identical to the Creative Commons Attribution Non-Commercial License (http://creativecommons.org/licenses/by-nc/4.0/). 


\section{Introduction}

Real-time convex probe endobronchial ultrasound-guided transbronchial needle aspiration (EBUS-TBNA) has become the standard of care for evaluating mediastinal and hilar lymphadenopathy ${ }^{1}$. Although EBUS-TBNA is a minimally invasive procedure with a high diagnostic yield, serious infectious complications such as mediastinitis, pericarditis, and sepsis have been reported ${ }^{2-7}$. Although rare, infectious complications can be life-threatening. We previously reported two cases of acute bacterial pericarditis after EBUS-TBNA, with one resulting in mortality ${ }^{8}$.

Recently our institution, a large tertiary referral hospital in South Korea, experienced two cases of mediastinal adenitis in patients with resectable lung cancer after EBUS-TBNA for mediastinal staging, which were initially misunderstood as lymph node metastasis on enhanced chest computed tomography scans (Figure 1). Although mediastinal adenitis was successfully managed by conservative treatment only, escalation of care was inevitable: prolonged hospital length of stay and operation time due to the presence of adhesions in the hilum in a patient with non-small cell lung cancer (Figure 1A-D), and prolonged hospital length of stay and a change in the treatment plan from surgery to chemoradiotherapy in the other patient with small cell lung cancer (Figure 1E-H).

Despite the occurrence of serious infectious complications after EBUS-TBNA, no consensus on any preventive strategies, including the use of prophylactic antibiotics, is available. Previous studies have suggested that the possible mechanism of infectious complications after EBUS-TBNA is the inoculation of oropharyngeal commensal bacteria into a target lesion by an aspiration needle ${ }^{9-11}$. When an EBUS bronchoscope passes through the oropharyngeal airway, the working channel of the scope can be contaminated by oropharyngeal commensal bacteria $^{7}$. Therefore, an aspiration needle inserted through this channel can also be contaminated.

We assume that oral hygiene care is important in preventing infectious complications during EBUS-TBNA. Chlorhexidine gluconate is a broad-spectrum antimicrobial agent, and chlorhexidine gluconate mouthrinse is simple, safe, and widely practiced $^{12}$. The aim of the present study is to investigate the effect of chlorhexidine mouthrinse on the prevention of microbial contamination during EBUS-TBNA.
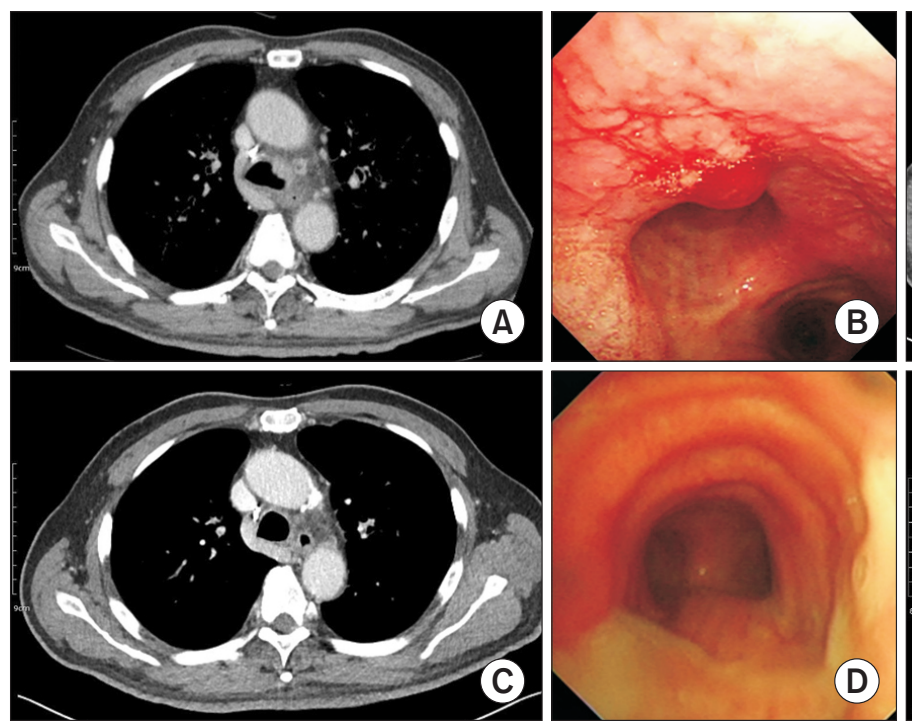
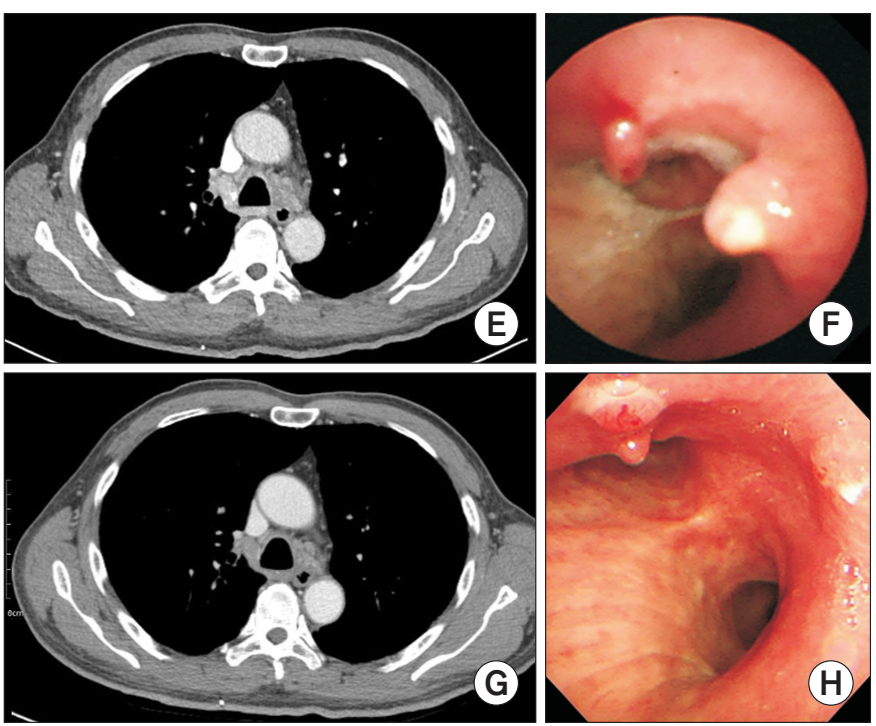

Figure 1. Two cases of mediastinal adenitis following endobronchial ultrasound-guided transbronchial needle aspiration (EBUS-TBNA). (AD) A 77-year-old man diagnosed with stage IIB (pT3N0M0) non-small cell lung cancer. (A) Chest computed tomography (CT) scan showed enlargement of the left lower paratracheal lymph node (station 4L) where EBUS-TBNA was performed 22 days previously and diffuse wall thickening of the adjacent trachea. (B) An inflammatory nodule and mucosal edema of the left lateral wall of the lower trachea were observed with flexible bronchoscopy on day 33 after EBUS-TBNA. Biopsy of the nodule revealed chronic active inflammation and fibrosis. (C) Chest CT scan on day 50; after antibiotic treatment, the lymph node decreased in size. (D) Bronchoscopic image on day 78; the inflammatory nodule and mucosal edema of the lower trachea had disappeared. (E-H) A 68-year-old man diagnosed with stage IA2 (cT1bN0M0) small cell lung cancer. (E) Chest CT scan showed enlargement of the lower paratracheal lymph nodes (station 4R and 4L) where EBUS-TBNA was performed 15 days previously and diffuse wall thickening of the adjacent trachea. (F) Inflammatory nodules and mucosal edema of the lower tracheal wall were observed by bronchoscopy on day 16 after EBUS-TBNA. (G) After antibiotic treatment, the lymph nodes decreased slightly in size, the inflammatory nodules and mucosal edema of the lower trachea were improving on day $26(\mathrm{H})$. 


\section{Materials and Methods}

\section{Study design and setting}

This study is a single-center, parallel-group, assessor-blinded randomized controlled trial (RCT) to be conducted at the Seoul National University Hospital in South Korea. Consenting and eligible participants who are scheduled to undergo EBUS-TBNA will be randomly assigned to either the intervention group or the control group at a 1:1 ratio. The flow diagram of this trial is shown in Figure 2. The protocol of this trial is described in accordance with the Standard Protocol Items: Recommendations for Interventional Trials (SPIRIT) guidelines ${ }^{13}$ (Figure 3).

\section{Eligibility criteria}

Hospitalized patients aged 19 years and older who are scheduled to undergo EBUS-TBNA using a convex probe will be included in this trial. Patients will be excluded if they meet any of the following criteria: (1) antiseptic mouthrinse within 7 days before inclusion; (2) active infection or antibiotic treatment within 7 days before inclusion; (3) being immunocompromised status; or (4) tracheostomy status. We will also exclude patients who have already undergone gastroscopy on the same day when EBUS-TBNA is scheduled to be administered.

\section{Topical anesthesia}

Our dedicated bronchoscopy suite is composed of a preprocedural room, two procedural rooms, and a disinfection and reprocessing room. The postprocedural area is the same as the preprocedural room. In the preprocedural room, all patients will gargle with $20 \mathrm{~mL}$ of $1 \%$ lidocaine for topical oropharyngeal anesthesia (Figure 4).

\section{Interventions}

Following topical oropharyngeal anesthesia, participants who are assigned to the intervention group will gargle for 1 minute with $100 \mathrm{~mL}$ of $0.12 \%$ chlorhexidine gluconate in the preprocedural room. Participants in the control group will not be provided with mouthrinse. The chlorhexidine mouthrinse intervention will be performed approximately 10 minutes before insertion of the bronchoscope (Figure 4).

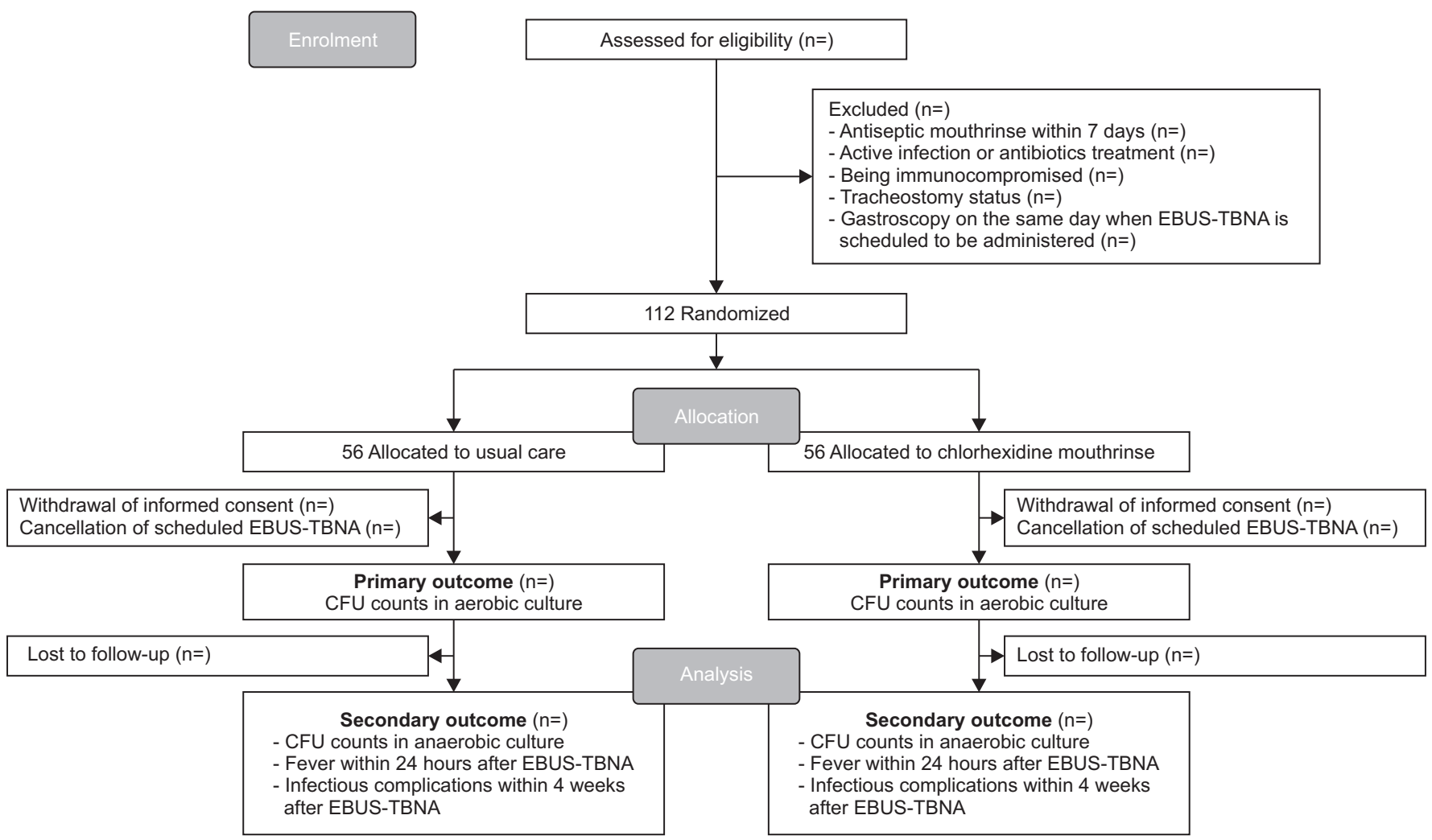

Figure 2. Flow diagram of the trial. CFU: colony forming unit; EBUS-TBNA: endobronchial ultrasound-guided transbronchial needle aspiration. 


\begin{tabular}{|c|c|c|c|c|c|c|c|c|c|}
\hline \multirow[b]{3}{*}{ Timepoint } & \multicolumn{9}{|c|}{ Study period } \\
\hline & \multirow{2}{*}{$\begin{array}{c}\text { Enrolment } \\
\begin{array}{l}-1 \text { day- } \\
\text { pre-allocation }\end{array}\end{array}$} & \multirow{2}{*}{$\begin{array}{c}\text { Allocation } \\
0\end{array}$} & \multicolumn{6}{|c|}{ Post-allocation } & \multirow{2}{*}{$\begin{array}{l}\text { Close-out } \\
4 \text { weeks }\end{array}$} \\
\hline & & & $\begin{array}{c}\text { Topical } \\
\text { anesthesia } \\
\text { by lidocaine }\end{array}$ & Sedation & $\begin{array}{l}\text { EBUS- } \\
\text { TBNA }\end{array}$ & $\begin{array}{c}1 \\
\text { day }\end{array}$ & $\begin{array}{c}2 \\
\text { days }\end{array}$ & $\begin{array}{l}2-4 \\
\text { days }\end{array}$ & \\
\hline \multicolumn{10}{|l|}{ Enrolment: } \\
\hline Eligibility screen & $\mathrm{x}$ & & & & & & & & \\
\hline Informed consent & $x$ & & & & & & & & \\
\hline Allocation & & $\mathrm{x}$ & & & & & & & \\
\hline \multicolumn{10}{|l|}{ Interventions: } \\
\hline Usual care (no mouthrinse) & & & $\bullet$ & $\longrightarrow$ & & & & & \\
\hline Chlorhexidine mouthrinse & & & $\bullet$ & $\longrightarrow$ & & & & & \\
\hline \multicolumn{10}{|l|}{ Assessments: } \\
\hline Baseline variables & $x$ & $x$ & & & $x$ & & & & \\
\hline \multicolumn{10}{|l|}{ Primary outcome } \\
\hline CFU counts in aerobic culture & & & & & & & $\mathrm{x}$ & & \\
\hline \multicolumn{10}{|l|}{ Secondary outcome } \\
\hline CFU counts in anaerobic culture & & & & & & & & $\mathrm{x}$ & \\
\hline Fever & & & & & & $\mathrm{x}$ & & & \\
\hline Infectious complications & & & & & & & & & $\mathrm{x}$ \\
\hline
\end{tabular}

Figure 3. Participant timeline. CFU: colony forming unit; EBUS-TBNA: endobronchial ultrasound-guided transbronchial needle aspiration.

\section{Implementation of EBUS-TBNA}

After participants move to a procedural room, conscious sedation will be initiated with intravenous midazolam and adjuvant fentanyl administered by an experienced nurse and titrated by the attending bronchoscopist. As the flexible bronchoscope is advanced orally, $1 \%$ lidocaine solution will be used for "spray-as-you-go" administration to the trachea. Prior to EBUS-TBNA, an endobronchial examination of the bronchial trees will be routinely performed using a flexible bronchoscope (BF-1T260, BF-Q290, BF-260, BF-P260F, Olympus, Tokyo, Japan) unless it has been performed within the previous several days. During the procedure, basic diagnostic sampling methods (bronchial wash, bronchoalveolar lavage, bronchial brush, transbronchial lung biopsy, and endobronchial biopsy) can be performed at the bronchoscopist's discretion. For EBUS-TBNA, an EBUS bronchoscope with a convex probe (BF-UC260FW, Olympus) will be inserted orally into the airway. Following identification of mediastinal or hilar lymph nodes by ultrasound, a 22-gauge aspiration needle (NA201SX-4022 or NA-U401SX-4022, Olympus) will be advanced through the working channel of the bronchoscope to puncture the airway wall at the predetermined position. Paratracheal and peribronchial lung masses can also be sampled. The bronchoscopist can replace the aspiration needle with a new one during the procedure. Transesophageal bronchoscopic ultrasound-guided fine needle aspiration (EUS-B-FNA) will be allowed. For EUS-B-FNA, the same EBUS bronchoscope will be re-inserted into the esophagus after the completion of EBUS-TBNA. A 22-gauge aspiration needle (NA-201SX-4022 or NA-U401SX-4022, Olympus) will be used for sampling through the esophageal wall (either the same needle used in EBUS-TBNA or a new one). The NA-201SX-4022 is usually used during the procedure; however, the U401SX-4022 can also be used, especially when the supply of NA-201SX-4022 from the manufacturer is insufficient. All bronchoscopic procedures including EBUS-TBNA will be performed by an experienced pulmonologist or a fellow in pulmonary and critical care medicine under the close supervision of the pulmonologist. As part of standard care, patients will be monitored by an experienced nurse under the direction of the bronchoscopist. Prescription of prophylactic antibiotics after EBUS-TBNA is allowed, and its use, type, dosage, and duration will be determined by the bronchoscopist or care provider. 
Secondary outcome: infectious complications
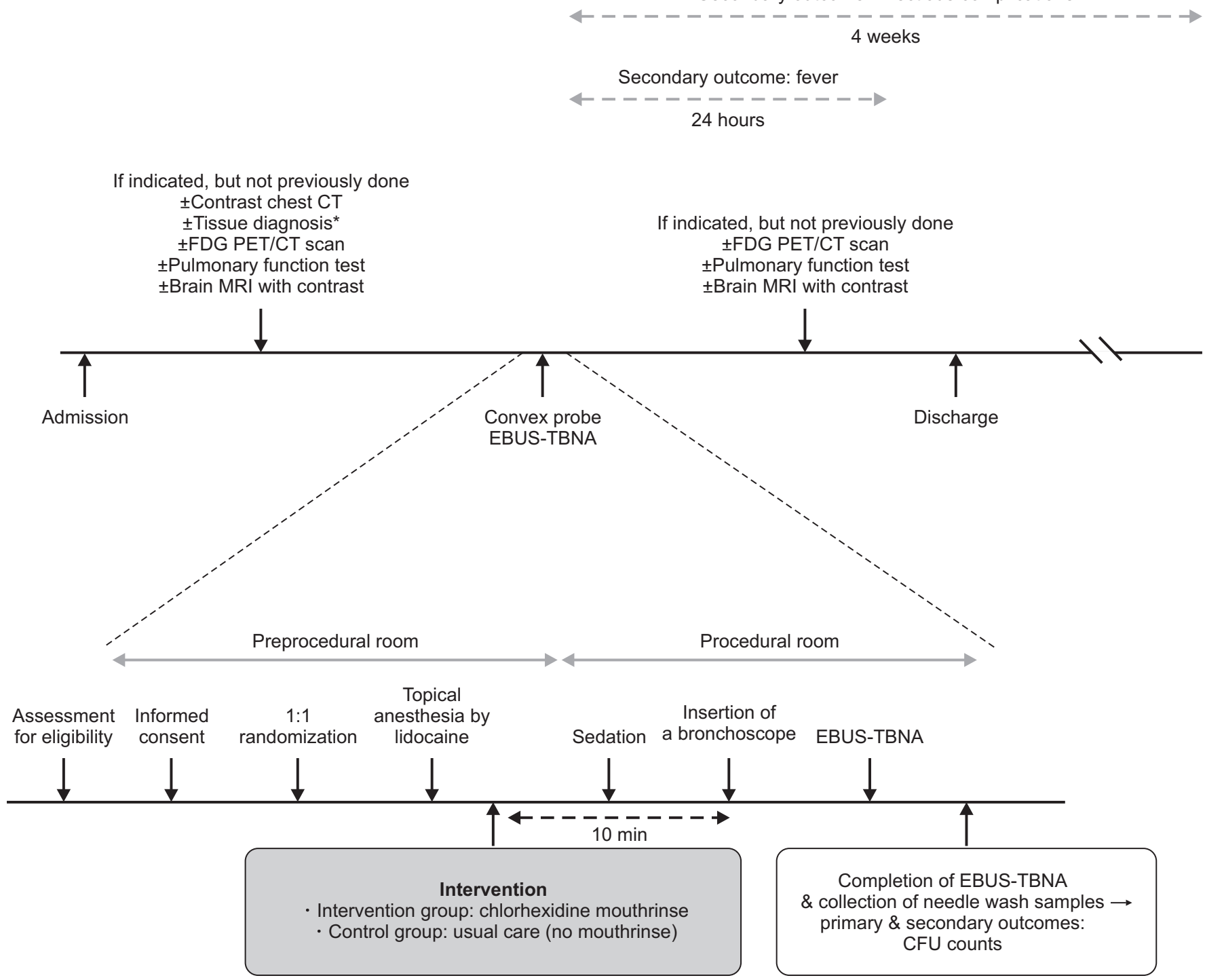

Figure 4. Overview of study procedures. *Tissue diagnosis can be done by transthoracic needle aspiration, radial probe EBUS, or diagnostic flexible bronchoscopy with endobronchial biopsy or transbronchial lung biopsy. CFU: colony forming unit; CT: computed tomography; EBUS-TBNA: endobronchial ultrasound-guided transbronchial needle aspiration; FDG: fluorodeoxyglucose; MRI: magnetic resonance imaging; PET: positron emission tomography.

\section{Microbial sample collection}

Immediately after completion of EBUS-TBNA on all targeted lesions with an aspiration needle and ejection of all aspirated materials from the needle for pathologic evaluation, a needle wash sample will be taken by instilling $5 \mathrm{~mL}$ of sterile physiological saline solution into the used needle, and collecting it in a sterile $10 \mathrm{~mL}$ test tube. This collection method has been reported previously ${ }^{10,14}$. If the bronchoscopist uses two or more needles during the procedure, each needle wash sample will be collected in each sterile tube immediately after completing the procedure. The samples will be immediately refrigerated at $5^{\circ} \mathrm{C}$ and processed on the same day.

Bacterial culture of the needle wash samples will be performed according to a routine clinical protocol. Needle wash samples will be plated on blood agar and chocolate agar plates, and incubated for 2 days under aerobic conditions. The samples will also be plated on Brucella agar plates, and incubated for 2-4 days under anaerobic conditions. If two or more needles are used per participant, each needle wash sample will be plated and incubated under both aerobic and anaerobic conditions. The bacteria will be identified by matrixassisted laser desorption ionization (MALDI) time-of-flight mass spectrometry using a microflex LT (Bruker Daltonics, 
Bremen, Germany). A colony will be placed on the target plate by the direct transfer method and analyzed with MALDI Biotyper RTC software 3.1 and the BDAL database (6903 MSP). A colony forming unit (CFU) will be measured according to the colony morphology and the result of identification.

\section{Outcomes}

The primary outcome is CFU counts in aerobic culture. If two or more needle wash samples are obtained in a participant, the primary outcome is the average of CFU counts in the aerobic plates. Secondary outcomes include CFU counts in anaerobic culture, fever within 24 hours after EBUS-TBNA, and infectious complications within 4 weeks after EBUSTBNA. If two or more needle wash samples are obtained in a participant, the average of CFU counts in the anaerobic cultures will be used as a secondary outcome measure. Fever is defined as a temperature $\geq 37.8^{\circ} \mathrm{C}$. Infectious complications include pneumonia, lung abscess, empyema, mediastinal adenitis/abscess, mediastinitis, pericarditis, and sepsis.

\section{Estimated sample size}

Sample size calculations are based on an available RCT of 100 patients who underwent gastroscopy with or without chlorhexidine mouthrinse due to a lack of similar previous studies on EBUS-TBNA ${ }^{15}$. To test whether chlorhexidine mouthrinse reduces the CFU counts of needle wash samples by $50 \%$ with a power of $90 \%$ and an alpha of 0.05 , the required sample size per group is 50 . Considering a potential dropout rate of $10 \%$, a sample size of 56 per group (112 in total) is required.

\section{Randomization}

A web-based randomization system has been implemented by the Medical Research Collaborating Center of the Seoul National University Hospital (https://mrcc.snuh.org/). A clinical research coordinator will perform randomization through the website. Each patient will be assigned an alphanumeric code. Investigators and other research personnel are unable to access the website. Only the clinical research coordinator is able to view intervention allocation on the website.

\section{Blinding}

The following personnel will be blinded to the intervention assignment: investigators including bronchoscopists performing EBUS-TBNA, technicians assisting with the procedure and instilling saline into aspiration needles to collect needle wash samples, outcome assessors including laboratory personnel, and care providers in general wards. Mouthrinsing with chlorhexidine will be done in the preprocedural room, which is separate from the procedural room, and bronchoscopists and assisting technicians in the procedural room can be blinded. However, participants are not blinded to the intervention assignment. Participants in the control group receive usual care (no mouthrinse). A placebo control such as pure water with or without a flavored additive will not be used in this trial because participants will be able to distinguish between chlorhexidine and placebos due to the odor and taste of chlorhexidine. Unblinding to an attending bronchoscopist performing EBUS-TBNA is possible if serious adverse events such as shock or seizure occur and if a relationship between the events and the chlorhexidine mouthrinse is suspected by the attending bronchoscopist. However, this unblinding has no consequence on the primary outcome measure.

\section{Data collection}

The below data will be collected.

\section{1) Patient factors}

(1) Demographic information: age, sex, body mass index, and smoking status

(2) The purpose for EBUS-TBNA: diagnosis and staging of malignancy or diagnosis of benign diseases such as tuberculous lymphadenitis and sarcoidosis

(3) Comorbidities: hypertension, diabetes mellitus, coronary heart disease, heart failure, stroke, chronic liver disease, chronic kidney disease, chronic obstructive pulmonary disease, and asthma

(4) Pathologic diagnosis

(5) Prescription of prophylactic antibiotics after EBUSTBNA, and its type, dosage, and duration

\section{2) Procedural factors}

(1) Bronchoscopic procedures other than EBUS-TBNA: bronchial wash, bronchoalveolar lavage, bronchial brush, transbronchial lung biopsy, endobronchial biopsy, and EUS-B-FNA

(2) The number of insertions of the EBUS bronchoscope

(3) The total number of lymph nodes punctured and the number of needle punctures per lymph node

(4) The number of aspiration needles used

(5) Characteristics of lymph nodes: size (less or more than $1 \mathrm{~cm}$ ), shape (oval or round), margin (indistinct or distinct), echogenicity (homogeneous or heterogeneous), and the presence of cyst, central hilar structure, coagulation necrosis sign, calcification, and pus-like discharge

(6) Procedure time, type and dosage of sedatives, attending bronchoscopists and their expertise

(7) Adverse events after chlorhexidine mouthrinse 


\section{Statistical analysis}

In all analyses, patients will be analyzed according to their randomization groups, with the exception of those who withdraw informed consent or do not undergo scheduled EBUS-TBNA (e.g., cancellation of scheduled EBUS-TBNA after successful diagnostic procedures such as endobronchial biopsy). CFU counts in the aerobic and anaerobic cultures of needle wash samples will be expressed as the mean \pm standard deviation and analyzed by the independent samples $t$ test if distributed normally. Otherwise, they will be described as the median (interquartile range) and analyzed using the MannWhitney U test. Fever within 24 hours after EBUS-TBNA and infectious complications within 4 weeks after the procedure will be presented as numbers and percentages and analyzed by either the chi-square test or Fisher exact test. Subgroup analysis according to the use of EUS-B-FNA and prophylactic antibiotics will be conducted. For the subgroup analysis according to prophylactic antibiotic use, only fever and infectious complications will be analyzed as outcomes. All comparisons will be two-sided and p-values of less than 0.05 will be considered statistically significant.

\section{Research ethics approval}

This study was approved by the Institutional Review Board of Seoul National University Hospital (H-2011-096-1173). This trial will be conducted in accordance with the principles of the Declaration of Helsinki and Good Clinical Practice. Written informed consent will be obtained from all participants before randomization.

\section{Confidentiality}

All data will be anonymized and identified by a coded ID number only to maintain participant confidentiality. All records containing personal identifiers such as informed consent forms will be stored separately from records identified by the coded ID number. All data will be stored in either a locked filing cabinet or in an electronic password-protected computer in a locked office. The access to data is restricted to authorized personnel only.

\section{Discussion}

EBUS-TBNA is a minimally invasive procedure; however, infectious complications following EBUS-TBNA can be fatal. To date, there have been limited data on preventive strategies to reduce infectious complications following EBUS-TBNA. We hypothesize that oral hygiene care has a role in preventing infectious complications after EBUS-TBNA.

Several studies have proposed that infectious complications after EBUS-TBNA are related to contamination of aspiration needles with oropharyngeal flora ${ }^{4,9-11}$. As the EBUS bronchoscope passes through the oral cavity and pharynx, the working channel of the scope can be contaminated by oropharyngeal flora. Microorganisms might be inoculated by puncture with the contaminated aspiration needle inserted through this channel. A previous study reported that contamination of aspiration needles with oropharyngeal commensal bacteria was relatively common-one-third of aspiration needle wash cultures were positive for typical oropharyngeal flora ${ }^{14}$. Moreover, a retrospective study showed that insertion of the EBUS bronchoscope through an endobronchial tube reduced contamination by oropharyngeal commensal bacteria-aspiration needle wash cultures were positive in only $3 \%$ of patients with endobronchial tubes, but in $100 \%$ of those without endobronchial tubes ${ }^{10}$.

Chlorhexidine mouthrinse is a promising candidate to prevent infectious complications following EBUS-TBNA. First, chlorhexidine gluconate has a broad spectrum of antimicrobial activity against gram-positive and gram-negative bacteria as well as yeast ${ }^{12}$. Second, oral hygiene care with chlorhexidine is widely practiced in critical care medicine because it reduces the risk of developing ventilator-associated pneumonia ${ }^{16}$. Third, a previous RCT in patients undergoing gastroscopy demonstrated that chlorhexidine mouthrinse reduced microbial CFU counts by $88 \%$ in endoscope samples ${ }^{15}$. Fourth, chlorhexidine mouthrinse is simple, safe, and cost-effective.

There are several risk factors other than contamination of the working channel or aspiration needle for developing infectious complications after EBUS-TBNA: the number of needle punctures per lymph node; the total number of lymph nodes punctured; the characteristics of lymph nodes (necrotic or cystic); and the expertise of the bronchoscopist ${ }^{7}$. These data will be collected in this trial.

This study will have limitations. Sample size estimation might be imprecise because it was based on an RCT in patients who underwent gastroscopy with or without chlorhexidine mouthrinse ${ }^{15}$. In addition, the primary outcome is a surrogate measure for the risk of infectious complications of EBUS-TBNA. If this trial proves the effectiveness of chlorhexidine mouthrinse in preventing microbial contamination during EBUS-TBNA, further large-scale studies will be required to assess outcomes such as the incidence of infectious complications following this procedure.

\section{Authors' Contributions}

Conceptualization: Kim NY, Cho J. Methodology: Kim NY, Park JH, Cho J. Formal analysis: Kim NY, Cho J. Enrollment of the patients: Kim NY, Park J, Kwak N, Choi SM, Park YS, Lee $\mathrm{CH}$, Cho J. Writing - original draft preparation: Kim NY, Park JH, Cho J. Writing - review and editing: Kim NY, Park JH, Cho J. 
Approval of final manuscript: all authors.

\section{Conflicts of Interest}

No potential conflict of interest relevant to this article was reported.

\section{Acknowledgments}

We thank Mi Ju Lee, RN, Nam Suk Lee, RN, Seon Mi Kim, Ji Won Kim, and Eun Jeong Jeon of our bronchoscopy suite for their assistance in performing the bronchoscopy. We also thank Seon Ho Um for laboratory assistance.

\section{Funding}

This study was supported by a 2020 Grant from the Korean Academy of Tuberculosis and Respiratory Diseases.

\section{References}

1. Vaidya PJ, Munavvar M, Leuppi JD, Mehta AC, Chhajed PN. Endobronchial ultrasound-guided transbronchial needle aspiration: safe as it sounds. Respirology 2017;22:1093-101.

2. Gu P, Zhao YZ, Jiang LY, Zhang W, Xin Y, Han BH. Endobronchial ultrasound-guided transbronchial needle aspiration for staging of lung cancer: a systematic review and meta-analysis. Eur J Cancer 2009;45:1389-96.

3. Asano F, Aoe M, Ohsaki Y, Okada Y, Sasada S, Sato S, et al. Complications associated with endobronchial ultrasoundguided transbronchial needle aspiration: a nationwide survey by the Japan Society for Respiratory Endoscopy. Respir Res 2013;14:50.

4. Fukunaga K, Kawashima S, Seto R, Nakagawa H, Yamaguchi $\mathrm{M}$, Nakano Y. Mediastinitis and pericarditis after endobronchial ultrasound-guided transbronchial needle aspiration. Respirol Case Rep 2015;3:16-8.

5. McGovern Murphy F, Grondin-Beaudoin B, Poulin Y, Boileau $\mathrm{R}$, Dumoulin E. Mediastinal abscess following endobronchial ultrasound transbronchial needle aspiration in a patient with sarcoidosis. J Bronchology Interv Pulmonol 2015;22:370-2.

6. Matsuoka K, Ito A, Murata Y, Sakane T, Watanabe R, Imanishi $\mathrm{N}$, et al. Severe mediastinitis and pericarditis after transbronchial needle aspiration. Ann Thorac Surg 2015;100:1881-3.

7. Voldby N, Folkersen BH, Rasmussen TR. Mediastinitis: a serious complication of endobronchial ultrasound-guided transbronchial needle aspiration. J Bronchology Interv Pulmonol 2017;24:75-9.

8. Lee HY, Kim J, Jo YS, Park YS. Bacterial pericarditis as a fatal complication after endobronchial ultrasound-guided transbronchial needle aspiration. Eur J Cardiothorac Surg 2015;48:630-2.

9. Epstein SK, Winslow CJ, Brecher SM, Faling LJ. Polymicrobial bacterial pericarditis after transbronchial needle aspiration: case report with an investigation on the risk of bacterial contamination during fiberoptic bronchoscopy. Am Rev Respir Dis 1992;146:523-5.

10. Minami D, Takigawa N, Oki M, Saka H, Shibayama T, Kiura K. Needle wash solution cultures following EBUS-TBNA with or without endobronchial intubation. Respir Investig 2018;56: 356-60.

11. Shimada S, Furusawa H, Ishikawa T, Kamakura E, Suzuki T, Watanabe Y, et al. Development of mediastinal adenitis six weeks after endobronchial ultrasound-guided transbronchial needle aspiration. Respir Med Case Rep 2018;25:161-4.

12. Jones CG. Chlorhexidine: is it still the gold standard? Periodontol 2000 1997;15:55-62.

13. Chan AW, Tetzlaff JM, Gotzsche PC, Altman DG, Mann H, Berlin JA, et al. SPIRIT 2013 explanation and elaboration: guidance for protocols of clinical trials. BMJ 2013;346:e7586.

14. Steinfort DP, Johnson DF, Irving LB. Incidence of bacteraemia following endobronchial ultrasound-guided transbronchial needle aspiration. Eur Respir J 2010;36:28-32.

15. Donatsky AM, Holzknecht BJ, Arpi M, Vilmann P, Meisner $\mathrm{S}$, Jorgensen LN, et al. Oral chlorhexidine and microbial contamination during endoscopy: possible implications for transgastric surgery: a randomized, clinical trial. Surg Endosc 2013;27:1914-22.

16. Hua F, Xie H, Worthington HV, Furness S, Zhang Q, Li C. Oral hygiene care for critically ill patients to prevent ventilatorassociated pneumonia. Cochrane Database Syst Rev 2016;10: CD008367. 